\title{
Acceptability of Two-Versus Three-Dose Human Papillomavirus Vaccination Schedule among Providers and Mothers of Adolescent Girls: A Mixed-Methods Study in Five Countries
}

\author{
Jessica Yasmine Islam ${ }^{\# 1}$, Alexis M. Hoyt ${ }^{\# 2}$, Silvina Ramos ${ }^{3}$, Karen Morgan ${ }^{4,5}$, Chan Joo \\ $\mathrm{Kim}^{6}$, Sylvia de Sanjose ${ }^{7}$, Nicole Butera ${ }^{8}$, Virginia Senkomago, PhD, MPH², Karin L. \\ Richter $^{9}$, Mary Anne McDonald ${ }^{10}$, Nadja A. Vielot, PhD, MSPH ${ }^{11}$, and Jennifer S. Smith, \\ PhD, MPH $^{1,12}$ \\ ${ }^{1}$ Department of Epidemiology, Gillings School of Global Public Health, University of North \\ Carolina, USA ${ }^{2}$ Department of Health Behavior, Gillings School of Global Public Health, \\ University of North Carolina, USA ${ }^{3}$ Centro de Estudios de Estado y Sociedad, Buenos Aires, \\ Argentina ${ }^{4}$ Perdana University School of Medicine, Malaysia ${ }^{5}$ Royal College of Surgeons, Dublin, \\ Ireland ${ }^{6}$ Department of Obstetrics and Gynecology, The Catholic University of Korea College of \\ Medicine, St. Paul's Hospital, Seoul, Korea ${ }^{7}$ Infections and Cancer, Cancer Epidemiology \\ Research Programme, Institut Català d'Oncologia, Spain ${ }^{8}$ Department of Biostatistics, Gillings \\ School of Global Public Health, University of North Carolina ${ }^{9}$ Department of Medical Virology, \\ University of Pretoria, National Health Laboratory Service, Pretoria, South Africa ${ }^{10}$ Center on \\ Genomics, Race, Identity, Difference, Duke University, Durham, NC, USA ${ }^{11}$ Department of Family \\ Medicine, School of Medicine, University of North Carolina, Chapel Hill, North Carolina, USA \\ ${ }^{12}$ UNC Lineberger Comprehensive Cancer Center, University of North Carolina, Chapel Hill, \\ North Carolina, USA \\ \# These authors contributed equally to this work.
}

\begin{abstract}
Purpose: The World Health Organization revised its HPV vaccination recommendations to include a two (2-) dose schedule for girls aged $\leq 15$ years. We investigated acceptability of 2-versus 3 -dose schedule among adolescent vaccination providers and mothers of adolescent girls in five countries.
\end{abstract}

Methods: Adolescent vaccination providers(N=151) and mothers of adolescent girls aged 9-14 years(N=118) were recruited from Argentina, Malaysia, South Africa, South Korea, and Spain. We assessed providers' preference for a 2-versus 3-dose HPV vaccination schedule via quantitative

\footnotetext{
Corresponding author: Jennifer S. Smith, PhD, MPH, Professor, Department of Epidemiology, UNC Gillings School of Global Public Health, 2103 McGavran Greenberg CB7435, Chapel Hill, North Carolina 27599, Fax: 919-966-2089, JenniferS@unc.edu.

Conflict of interest

The remaining authors have no conflicts of interest to disclose.

Trademarks

Cervarix is a trademark owned by or licensed to the GSK group of companies.
} 
surveys. Mothers' attitudes toward a 2-dose schedule were assessed through focus group discussions.

Results: Most adolescent providers preferred a 2-over 3-dose HPV vaccination schedule (overall: $74 \%$ ), with preference ranging from $45.2 \%$ (South Africa) to $90.0 \%$ (South Korea). Lower cost, fewer clinic visits, and higher series completion were commonly-cited reasons for 2-dose preference among providers and mothers. Safety and efficacy concerns were commonly-cited barriers to accepting a 2-dose HPV vaccination schedule among providers and mothers. Mothers generally accepted the reduced schedule, however, requested further information from a trusted source.

Conclusions: Adolescent vaccination providers and mothers preferred the 2-dose over 3-dose HPV vaccination schedule. Acceptability of a 2-dose HPV vaccination could be improved with additional information to providers and mothers on HPV vaccination safety and efficacy.

\section{Keywords}

vaccination; acceptability; human papillomavirus; dose; adolescent; providers; parents; focusgroups

\section{INTRODUCTION}

Globally, human papillomavirus (HPV) is the most common sexually transmitted infection and has been established as the cause of cancers of the cervix, anus, vagina, vulva, penis, and oropharynx[1]. Highly effective prophylactic vaccines have been developed to prevent HPV persistence and HPV-associated cancers in women and men. Since 2006, HPV vaccination has been introduced globally into national immunization programs for girls in 71 countries, and for boys in 11 countries[2]. Countries with national immunization programs have seen varied national coverage of HPV vaccination due to programmatic barriers including cost, lack of knowledge of HPV and cervical cancer, vaccination safety concerns, and dose completion difficulties for patients and providers [3-6]. Programmatic barriers of HPV vaccination uptake are of particular concern in low- and middle-income countries (LMICs), with the greatest global burden of HPV-related cancers, particularly cervical cancer[7].

Offering an HPV vaccination schedule with fewer doses is one promising approach to reduce barriers to vaccination. Clinical trials have demonstrated that a 2-dose schedule elicits and sustains non-inferior immune responses in adolescent girls aged $<15$ years old as compared with the 3-dose regimen, over a five-year time period [8-12]. In 2014, the World Health Organization (WHO) Strategic Advisory Group of Experts (SAGE) revised its HPV vaccination recommendation in accordance with emerging scientific evidence, and now globally recommends adolescents under 15 years of age receive a 2-dose schedule, with 3doses still recommended for adolescents 15 years and older and the immunocompromised or infected with human immunodeficiency virus (HIV)[13]. Transitioning to a 2-dose schedule has the potential to increase HPV vaccination coverage at a reduced cost if efforts are focused on vaccinating the WHO recommended target group. Availability of a 2-dose 
schedule presents an important opportunity to substantially reduce programmatic barriers to providing HPV vaccination.

Mothers are usually the primary health care decision maker for their children[14]. A mother's HPV vaccination intention for her adolescent child is influenced by several factors including her attitudes towards HPV vaccination, perceptions of community opinion regarding HPV vaccination, and perceived difficulty of getting HPV vaccination[15]. These intentions are further influenced by health care providers. High quality provider recommendation is the primary influencer of HPV vaccination initiation among mothers for their adolescents and is positively correlated with higher rates of HPV vaccination in adolescent girls [16-19].

Previous studies have evaluated HPV vaccination acceptability in parents of eligible adolescents and health care providers [5,20-23]. However, to our knowledge, no research study has assessed provider and maternal perceptions of a 2-versus 3-dose HPV vaccination schedule across countries using a standardized questionnaire. Further data are needed on providers' and mothers' acceptance of a lower dose HPV vaccination schedule to inform the global implementation of HPV vaccination programs for adolescents, particularly as prior studies have identified knowledge of HPV vaccination efficacy and safety as a major barrier to three-dose HPV vaccination uptake among adolescents [24].

Our primary aim was to solicit provider and mother opinions of the acceptance of a 2-dose HPV vaccination schedule in five countries, including Argentina, Malaysia, South Africa, South Korea, and Spain. We included distinct geographical continents to inform global implementation of 2-dose HPV vaccination and invited international investigators globally known to the HPV field. Selected countries included a variety of contexts for HPV vaccination availability. Argentina, Malaysia, and Spain expanded their respective National Immunization Programs to cover the cost of HPV vaccination for female adolescents in 2011, 2010, and 2007 respectively, prior to this study. However, South Africa and South Korea did not have a national program covering the cost of HPV vaccination at the time this survey was completed. Here, we present data on the acceptability of a 2-versus 3-dose HPV vaccination schedule among both adolescent health care providers and mothers of adolescent girls. Data from in-country providers contributes new insights into HPV vaccination acceptability in countries with little or no existing research on provider perceptions of the updated 2-dose schedule of HPV vaccination.

\section{METHODS}

\section{Study Participants}

Providers(Quantitative): We implemented a cross-sectional survey of health care providers who administer vaccination to adolescents, hereafter referred to as adolescent vaccination providers. The survey was conducted in five countries from diverse geographical and sociocultural regions, including Argentina, Malaysia, South Africa, South Korea, from October 2013 to April 2014 Study staff identified eligible providers with the authority to administer adolescent vaccines by examining each country's medical regulations. Further site-specific details on recruitment of providers are available in Appendix 1. Providers were 
eligible to participate if they actively administered adolescent vaccines. Adolescent vaccination providers were identified via non-probability convenience sampling and recruited through mail, email, phone, or in-person. Participating providers were compensated for their time and associated travel required for the study interview.

Mothers (Qualitative): Four focus group discussions were conducted in each country between November 2013 and April 2014, including at least one focus group with mothers of vaccinated daughters and at least one focus group with mothers of unvaccinated daughters, except Spain where six mothers of unvaccinated daughters completed individual semistructured interviews rather than focus group discussions. Given high HPV vaccination coverage in Spain, local investigators were unable to efficiently recruit enough participants who had not vaccinated their daughters to participate in focus group discussion format. To address this concern, the Spain study site conducted 6 individual interviews with women who had not vaccinated their daughters. Mothers were recruited using non-probability convenience sampling from medical offices, health centers, or schools (Appendix 1). To be eligible, mothers had to have a daughter old enough to receive HPV vaccination, based on each country's vaccination guidelines at the time of data collection up to 14 years of age (age: 9 years in South Africa; 11 years in Argentina/South Korea/Spain; 13 years in Malaysia). Eligible, interested mothers were stratified into focus groups based on whether their daughter was either (i). vaccinated or (ii). unvaccinated. A total of 124 mothers (70 with vaccinated daughters, 54 with unvaccinated daughters) were enrolled across the five countries (Argentina [ $\mathrm{n}=23]$; Malaysia [ $\mathrm{n}=26]$; South Africa $[\mathrm{n}=21]$; South Korea $[\mathrm{n}=$ 31]; and Spain $[n=23]$ ), with a median of 5.5 participants per focus group.

Study collaborators in each country secured Institutional Review Board (IRB) approval from their respective institutions prior to data collection. UNC study staff received IRB approval for analysis of de-identified secondary data.

\section{Measures}

Providers (Quantitative): Surveys were administered in-person or by phone to providers. A trained study interviewer collected information on providers' demographic information and experiences administering HPV vaccination to adolescent girls within the age guidelines for each country. Providers were given different questionnaires based on whether they had previously administered HPV vaccination. A total of eight questions assessed attitudes towards willingness for initiation and for completion of a 2-versus 3-dose HPV vaccination schedule, as well as anticipated successes and barriers to a 2-dose schedule (see Appendix 2). The response to the following question was used as primary measure of preference for the 2-dose HPV vaccination, i.e. the outcome for multivariable regression analyses: "If a 2dose HPV vaccine were to become available, which would you recommend more frequently: A 3-dose HPV vaccine or a 2-dose HPV vaccine?" Country study staff entered de-identified data into English language EpiData forms, translating data into English when necessary.

Mothers (Qualitative): Each focus group discussion followed a semi-structured script that assessed mothers' attitudes towards various aspects of adolescent HPV vaccination, including questions to evaluate knowledge of cervical cancer, willingness to obtain the HPV 
vaccination for their daughters, barriers and facilitators to obtaining the HPV vaccination for their daughters, and efficacy of a 2-dose schedule. The present analysis pertains to focus group discussions around maternal attitudes towards a 2-dose compared to 3-dose HPV vaccination schedule. Focus group moderators asked questions from a discussion guide, and additional questions emerged through probing and clarifying statements (see Appendix 3).

\section{Analysis}

Providers (Quantitative)—Data obtained from providers were double-entered in English by staff in each country as a measure for quality control of data. Identified discrepancies were resolved in consultation with each site's primary investigator. A final de-identified data set was sent to UNC for final data cleaning and analysis. Univariate analyses were conducted across country sites to assess baseline characteristics of providers and mothers of adolescents, and to evaluate adolescent vaccine practices and attitudes among providers. Multivariable logistic regression was utilized to identify predictors of 2-versus 3-dose preference for HPV vaccination. Models were fit to estimate multivariable-adjusted odds ratios (aORs) and associated 95\% confidence intervals (CIs) for the following predictor variables separately, adjusted for country of origin: type of provider; providers' years of practice; whether provider administered HPV vaccination; provider's average number of adolescent female patients seen per month; providers' perceptions of efficacy of 2-dose HPV vaccination; and provider's perceptions of potential side effects of the 2-dose as compared to 3-dose HPV vaccination. All quantitative analyses were conducted in SAS 9.3 (SAS Institute Inc., Cary, NC).

Mothers (Qualitative)—In-country researchers facilitated the verbatim transcription of focus group discussion recordings and their translation into English. Translations were reviewed by in-country study staff who were native speakers of the language used during the focus group discussion and fluent in English. UNC study staff reviewed the translations for meaning, and clarified colloquial usages, local references and cultural contexts, with the lead in-country researchers before analysis, and during analysis as needed.

An experienced qualitative researcher (McDonald) supervised data management and analysis, which includes the following procedures. Cleaned transcripts were entered into ATLAS.ti (ver. 7, Berlin, Germany) for thematic analysis[25,26]. We began by using a structured codebook based on the study aim and the focus group discussion script. A thematic analysis framework was used to code the data. Additional codes were added as they emerged from the data through an iterative process: one focus group discussion transcript from each country was then reviewed independently by two research assistants and coded, along with additional emergent themes. Next, these transcripts were compared and inconsistent coding decisions were discussed until a final agreement was reached. Additional emergent codes were incorporated into the codebook, and remaining transcripts were coded and reconciled using the same procedure [27-30]. Below, qualitative data are summarized thematically. 


\section{RESULTS}

\section{Providers (Quantitative Analysis)}

Providers' Medical Practice-Of 353 providers contacted, 151 were enrolled across the five countries (Argentina [n=30]; Malaysia [ $n=30]$; South Africa [n=31]; South Korea $[\mathrm{n}=30]$; and Spain $[\mathrm{n}=30])$. The most common practitioner types were family, internal, or general practitioners (31\%); obstetrics/gynecologists (OB/GYN) (26\%); and pediatricians (22\%) (Table 1). All adolescent providers from Argentina (100\%), and most from Spain $(87 \%)$ and Malaysia (77\%) had provided HPV vaccination to adolescents, compared with half in South Korea (50\%) and about one-third in South Africa (36\%) (Table 2).

\section{Providers' Attitudes towards a 2-Versus 3-dose HPV Vaccination Schedule-} Most providers (75\%) reported that they would recommend a 2-dose compared to 3-dose schedule, ranging from 45 in South Africa to $90 \%$ in South Korea (Figure 1). Most common reasons for recommending a 2-dose vaccine more frequently to parents of adolescent girls were lower cost (Argentina: 27\%; Malaysia: 53\%; South Africa: 16\%; South Korea: 60\%; Spain: $47 \%)$, fewer office visits $(10 \%, 30 \%, 19 \%, 67 \%, 70 \%$ respectively), higher series completion $(67 \%, 3 \%, 19 \%, 50 \%, 63 \%$ respectively), and less pain $(37 \%, 10 \%, 10 \%, 33 \%$, $53 \%$ respectively) (Figure 2).

About one-third of providers noted that cost was the main barrier to HPV vaccination that a 2-dose schedule could address, ranging from $10 \%$ in Spain to $57 \%$ in Malaysia (Table 2). Fewer clinic appointments were cited as a main advantage of a 2-dose HPV vaccination schedule, ranging from $23 \%$ in Malaysia to $83 \%$ in Spain. When asked specifically about potential concerns of a 2-dose HPV vaccination schedule, providers cited concerns regarding the possibility of lower vaccine efficacy (54\%), ranging from $10 \%$ of providers in South Africa to $77 \%$ in South Korea. Several providers stated they would need more information on the efficacy of the 2-dose HPV vaccination (61\%), ranging from 19\% of providers in South Africa to $87 \%$ in South Korea.

Fewer than half of providers from South Africa preferred the 2-dose HPV vaccination $(45 \%)$. Additionally, we observed an increase in the proportion of 2-dose preference of HPV vaccination among providers with 20 female adolescent patients or more (79\%), as compared to providers with $<20$ per month $(70 \%)$ (Table 3$)$. Adolescent vaccination providers were more likely to prefer the 2-dose than the 3-dose HPV vaccination schedule if they believed that 2-dose HPV vaccination is as effective as a 3-dose vaccination schedule (adjusted OR: 8.25, 95\% CI: 3.09-22.03) (Table 3). Provider preference for a 2-versus 3dose vaccination schedule did not appear to be associated with any other factor examined, including type of provider, provider years of practice, HPV vaccination provision status, average number of adolescent girls seen in provider's clinic per month, and provider's opinion of whether the 2-dose HPV vaccination schedule would have more side effects than 3-dose vaccination. 


\section{Mothers of adolescent daughters (Qualitative Analysis)}

Qualitative data provided insight into mothers' rationale regarding their preferences for the 2-versus 3-dose HPV vaccination. The focus group discussions revealed a diversity of mothers' understanding of the basics of vaccination and the immunization process, the HPV virus, and the etiology of cervical cancer across the five countries and within focus groups (data not shown). Table 4 describes emergent themes that, while seen to some degree in all groups, were salient to each country group. Focus group discussions with mothers of unvaccinated girls voiced similar perceived advantages and concerns to those of unvaccinated girls, with slight exceptions. Many themes related to the 2-versus 3-dose HPV vaccination were consistent across focus groups.

\section{Perceived Advantages of a 2-Versus 3-dose HPV Vaccination Schedule}

Convenience: Most women recognized that a 2-dose HPV vaccination schedule would be easier to complete, and would be more convenient and timesaving compared to a 3-dose schedule.

"I think that two doses, because of some mothers that sometimes don't take them [daughters], they already have a hard time taking them to the outpatient clinic. ... It would be easier for them." - Argentina Vaccinated Focus Group

"Just as if they want to make a one-dose vaccine, even better, you think, if it covers just the same and you only have to get shot once, great!" - Spain, Vaccinated Focus Group

However, other mothers said that the convenience afforded by the 2-dose was only acceptable if they were sure it provided "protection" as strong as that of the 3-dose HPV vaccination.

"Then twice is way better. Under the assumption that they have same efficacy, twice is more convenient." - Korea, Unvaccinated Focus Group

Favorable attitude towards scientific innovation: Many mothers across countries rationalized that the change in dosing schedule is based on new research and scientific innovation. These mothers positively associated scientific innovation with the updated 2dose vaccination schedule.

"That increases my reliability [how reliable she thinks the vaccine is] since it seems like the improved version, like Samsung introducing newer version - South Korea, Unvaccinated Focus Group

"There must be a reason. If the reason is good, then we have to accept it. Because the research has been done. If the evidence states that 2 is sufficient we have to follow." - Malaysia, Vaccinated Focus Group

\section{Perceived Barriers to a 2-Versus 3-dose Schedule}

Concerns about the strength of vaccination: Across country sites, concerns surfaced regarding the efficacy of the HPV vaccination if it were reduced from three to two doses. Some mothers assumed that the 2-dose schedule would include the same amount of drug as 
the 3-dose schedule in fewer injections, which increased concerns about side effects, both short term and long term.

"Okay, because maybe then the dose is much larger and there might be more side effects and such, right? Like, we also have to take that into account. Putting three doses in one." - Spain, Vaccinated Focus Group

"No. In my case, I would ask the pediatrician about two or three times, really. Because if it is three doses, and then it's only two, they would be stronger, 11 years old and such a strong vaccination, I don't think so.” - Argentina, Vaccinated Focus Group

Cost: Like providers, cost was an important consideration for mothers' decision making.

"Before I have to get the full information as to why is the different between the 3doses and the 2-doses before I can; and I have to know why they are different and why the other one is cheaper and why the other one is expensive before I will be able to say to other people which one to take." - South Africa, Unvaccinated Focus Group

Participating countries had different national health care payment systems, and socioeconomic status varied considerably across participants, making it difficult to compare responses regarding cost among focus groups. For example, in Argentina, HPV vaccination is part of the mandatory vaccination policy for adolescents and is free of cost. Mothers in South Korea and South Africa attributed new recommendations with effectiveness, noting that even if the 2-dose was more expensive, they would still choose it.

"I will have it [the two dose] if it's much better than the previous one even though it's more expensive..." - South Korea, Unvaccinated

"When it comes to prevention the cost comes last, cost should not be an issue. As long as you are preventing, cost should not be an issue." - South Africa, Vaccinated Focus Group

\section{Information Needed to Accept a 2-dose Vaccination Schedule}

Desire for reliable information from trusted sources: Mothers from both focus group discussion groups (with vaccinated and unvaccinated daughters) expressed interest in receiving individualized, concise information from a trusted provider. Mothers generally respected the opinions of doctors and researchers, however, they expressed interest in receiving consistent, reliable information first-hand.

"...the truth is that we are not thoroughly informed because there was no campaign. There is a campaign to vaccinate, but not one to inform..." - Argentina, Vaccinated Focus Group

Most women wanted to make sure they had all information to make the right decision. Interestingly, after hearing about the 2-dose HPV vaccination, some mothers of vaccinated daughters questioned their decision to vaccinate their daughter with the 3-dose schedule. 
"When they make the change, they don't tell you why. Then we don't know whether it is better or if what we did was wrong, if it is better now." - Argentina Vaccinated Focus Group

Many mothers did not understand what HPV vaccination protected against, or the associated side effects, which coincided with lower knowledge about HPV vaccination and cervical cancer. All focus group discussions included varying degrees of knowledge of the efficacy and potential side effects of HPV vaccination.

"If the effect is the same, then, I don't know, I don't know how vaccines work or don't." - Spain, Vaccinated Focus Group

\section{Discussion}

In this mixed-methods study of five countries, adolescent health care providers and mothers of adolescent girls tended to prefer a 2-dose over a 3-dose HPV vaccination schedule. Providers cited that the 2-dose schedule addressed many barriers of the 3-dose schedule, with the potential for lower cost, fewer clinic visits, higher series completion, and less patient pain. Mothers generally preferred a reduced HPV vaccination schedule, citing greater convenience and favorable attitudes towards scientific innovation. Both providers and mothers, however, expressed some concerns and the need for more information regarding the relative safety and efficacy of 2-dose compared to 3-dose HPV vaccination. Our study findings are consistent with previous research suggesting that a 2-dose regimen can effectively address barriers to 3-dose HPV vaccination[31,32], including cost of HPV vaccination Two-dose HPV vaccination has been shown to be more cost-effective when compared to 3-dose HPV vaccination in both the short- and long-term .

Provider recommendation is the primary predictor of HPV vaccination uptake[33]. The positive association between awareness of HPV vaccination, and HPV vaccination initiation and completion among parents of adolescents has been shown to depend on strong provider recommendation[34,35]. Providers across countries in our study had varying degrees of understanding of whether the efficacy and safety of a 2-dose HPV vaccination schedule was comparable to a 3 -dose schedule. Previous studies have found that providers do not regularly recommend HPV vaccination for a several reasons, including ambiguity regarding who and what age group needs to receive the vaccine, and confidence in addressing parental concerns[36,37]. In our study, while providers generally supported a 2-dose HPV vaccination schedule, they had concerns regarding its efficacy and safety, and requested further information on these topics to feel more comfortable for their patient recommendations.

Like providers, mothers were generally accepting of a 2-dose HPV vaccination due to greater convenience and reduced cost. However, several mothers requested more information regarding efficacy and safety before making a confident decision. Our focus group findings echo previous research regarding mothers' attitudes towards a 3-dose schedule. Qualitative research conducted among women in South Africa and Ohio Appalachia highlighted the importance of mother and female caretakers in health decision making for the initiation of the 3-dose HPV vaccination for their daughters; support for HPV vaccination was observed, 
with concurrent concerns related to the vaccination's cost and side effects[14]. This simultaneous support and concern of HPV vaccination is reflected in other studies in LMICs related to the 3-dose schedule [38-42]. In the current study, concerns related to cost had the potential to be addressed by a 2-dose schedule, although concerns related to resulting changes in vaccine efficacy and associated side effects remained to varying degrees by country.

Notable variability in knowledge of both providers and parents has been identified in a global systematic review that described WHO's SAGE definitions of vaccination hesitancy as influenced by several factors including confidence, complacency, and convenience[43]. This SAGE review noted that factors related to vaccination hesitancy varied widely by country, and were further nuanced by social and cultural norms [43]. A systematic review of Sub-Saharan Africa similarly showed high levels of willingness and acceptability of the 3dose HPV vaccination among adolescent girls, parents of adolescents, and health care workers while identifying notable gaps in knowledge and education regarding HPV, HPV vaccination, and cervical cancer among these same groups.

Study advantages included the use of a standardized mixed methods design across five geographically diverse sites, which allowed for better understanding of similarities and differences in acceptability of 2-dose HPV vaccination schedule across countries. While many LMICs have made successful transitions from a 3-to 2-dose HPV vaccination schedule, social mobilization and acceptability remain important communication strategies to promote uptake, particularly through face-to-face interactions[44]. Since HPV vaccination perceptions and knowledge differed by country, messaging and educational campaigns for a 2-dose vaccination schedule will need to be tailored to the specific social, economic, and political climate of the region of introduction. This approach is supported by previous research investigating various means of achieving high HPV vaccination coverage in LMICs[5], as well as studies conducted in high-income countries that recognize the need for multiple strategies for vulnerable population sub-groups.

Limitations of this study included a relatively small provider sample size and nonprobability based sampling strategy of providers, limiting the generalizability of the findings within these countries. Small sample sizes of providers and mothers precluded the use of statistical tests to assess group differences between countries, or between mothers of vaccinated and unvaccinated daughters. Additionally, due to small sample size, we were unable to account for clustering by country in our quantitative analysis. With larger sample sizes by country, we may have observed significant odds ratios within logistic regression analyses. Given that we utilized convenience sampling to recruit mothers of adolescents, mothers who participated in focus group discussions were more often from urban areas, and thus likely with greater access to medical providers and health clinics. In our study, we did not directly interview adolescents regarding their opinions of HPV vaccination as mothers are usually the primary health care decision makers for their children and due to issues regarding consent for adolescent participants. Finally, given that the focus group guide was based on prior literature of barriers to HPV vaccination, we acknowledge that this approach was more pragmatic than a ground-up, inductive approach to elicit participants' potential concerns about HPV vaccination. 
Our findings indicate that both providers and parents could benefit from focused education regarding a 2-dose HPV vaccination schedule. While some logistical barriers to HPV vaccination acceptance are addressed by a reduced 2-dose vaccination schedule, concerns related to potential for increased side effects and lower efficacy seem to remain, despite general acceptability of HPV vaccination. Though mothers' acceptability of a 2-dose HPV vaccination schedule varied by country site, this acceptability could be enhanced by additional information regarding safety and efficacy by providers. These findings underscore the importance of a strong health provider recommendation for a 2-dose HPV vaccination to bolster mothers' acceptance of the vaccination for their daughters, including opportunities to address any concerns related to side effects and efficacy. A strong recommendation with patient education from a trusted healthcare provider has been shown to be a primary vaccination mediator among parents of adolescents [16-19]. Evidence from the current study supports this finding for a 2-dose HPV vaccination schedule. Providers generally accepted a 2-dose schedule, although but it is unknown how strong their recommendations would be as knowledge about safety and efficacy varied across sites.

Future implementation science research should be focused on equipping providers with the knowledge and optimal communication skills to improve HPV vaccination discussions with parents from varying socioeconomic and education levels. Literature from high-income countries has suggested that HPV vaccination availability and promotion alone is not enough to influence significant uptake, and identified the need for comprehensive approaches that target multiple levels of the socioecological model [45-47] in culturally responsive ways[14].

Reducing the HPV vaccination dosing schedule from 3-to 2-doses has the potential to reduce logistical barriers for patients and providers. This, combined with increased access and culturally relevant communication campaigns that promote high-quality recommendations from providers can effectively increase vaccination rates in LMICs and reduce the burden of HPV-related cancers for future generations. Knowledge generated from this study can inform future programmatic decisions as more countries adopt a 2-dose vaccination schedule, and potentially a 1-dose schedule for HPV vaccination, if recommended based on currently evolving scientific evidence.

\section{Supplementary Material}

Refer to Web version on PubMed Central for supplementary material.

\section{Acknowledgments}

The authors gratefully acknowledge the participants of this study. The authors thank Suzanne Landi, Shoshana Goldberg, and Sara Smith for their assistance in developing this manuscript. JYI was supported by a NIH NRSA individual predoctoral fellowship (F31-CA210474-01A1).

Funding

This study was funded by GlaxoSmithKline Biologicals SA (Study ID: 117339); all data collection, analysis and interpretation was conducted by study co-authors, and GSK had the opportunity to review this paper. GSK did not have input into the research data collection, analysis, or interpretation of results. 
Jennifer S. Smith has received research grants, served on paid advisory boards, and/or been a paid speaker for GSK group of companies and Merck \& Co., Inc. over the past five years.

\author{
Abbreviations \\ HIV \\ human immunodeficiency virus \\ HPV \\ human papilloma virus \\ LMIC \\ low and middle income country \\ SAGE \\ Strategic Advisory Group of Experts \\ STI \\ sexually transmitted infections \\ WHO \\ World Health Organization
}

\title{
References
}

1. Human papillomavirus vaccines: WHO position paper (2014). World Health Organization, Geneva, Switzerland

2. Human papillomavirus vaccines: WHO position paper, May 2017 (2017). World Health Organization, Geneva, Switzerland

3. Clendinen C, Zhang Y, Warburton R, Light D (2016) Manufacturing costs of HPV vaccines for developign countries. Vaccine 34 (48):5984-5989 [PubMed: 27771183]

4. Remes P, Selestine V, Changalucha J, Ross D, Wight D, de Saniose S, Hayes R, Watson-Jones D (2012) A qualitative study of HPV vaccine acceptability among health workers, teachers, parents, female pupils, and religious leaders in northwest Tanzania. Vaccine 30 (36):5363-5367 [PubMed: 22732428]

5. LaMontagne D, Barge S, Le N, Mugisha E, Penny M, Gandhi S, Janmohamed A, Kumakech E, Mosqueira N, Nguyen N, Paul P, Tang Y, Minh T, Uttekar B, Jumaan A (2011) Human papillomavirus vaccine delivery strategies that achieved high coverage in low- and middle-income countries. Bull World Health Organization 89 (11):821-830B

6. Brewer N, Fazekas K (207) Predictors of HPV vaccine acceptability: a theory-informed, systematic review. Prev Med 45 (2-3):107-114 [PubMed: 17628649]

7. Wigle J CE, Watson-Jones D (2013) Human papillomavirus (HPV) vaccine implementation in low and middle-income countries (LMIC): Health system experiences and propects. Vaccine 31 (37): 3811-3817 [PubMed: 23777956]

8. Lazcano-Ponce E, Stanley M, Muñoz N, Torres L, Cruz-Valdez A, Salmerón J, Rojas R, Herrero R, Hernández-Ávila M (2014) Overcoming barriers to HPV vacination: non-inferiotity of antibody response to human papillomavirus $16 / 18$ vaccine in adolescents vaccinated with a two-dose vs. a three-dose schedule at 21 months. Vaccine 32 (6):725-732 [PubMed: 24355090]

9. Dobson S, McNeil S, Dionne M, Dawar M, Ogilvie G, Krajden M, Sauvageau C, Scheifele D, Kollmann T, Halperin S, Langley J, Bettinger J, Singer J, Money D, Miller D, Naus M, Marra F, Young E (2013) Immunogenicity of 2 doses of HPV vaccine in younger adolescents vs 3 doses in young women: a randomized clinical trial. JAMA 309 (17):1793-1802 [PubMed: 23632723]

10. Romanowski B, Schwarz T, Ferguson L, Peters K, Dionne M, Schulze K, Ramjattan B, Hillemanns P, Catteau G, Dobbelaere K, Schuind A, Descamps D (2011) Immunogenicity and safety of the HPV-16/18 AS04-adjuvanted vaccine administered as a 2-dose schedule compared with the licensed 3-dose schedule: results from a randomized study. Human Vaccine 7 (12):1374-1386

11. Romanowski B, Schwarz T, Ferguson M, Klaus P, Dionne M, Schulze K, Ramjattan B, Hillemanss P, Behre U, Suryakiran P, Thomas F, Struyf F (2014) Immune response to the HPV-16/18 AS04adjuvanted vaccine administered as a 2-dose or 3-dose schedule up to 4 years after vaccination. Human Vaccines \& Immunotherapeutics 10 (5):1155-1165 [PubMed: 24576907] 
12. Romanowski B, Schwarz T, Ferguson L, Peters K, Dionne M, Behre U, Hillemanns P, Suryakiran P, Thomas F, Struyf F (2016) Sustained immunogenicity of the HPV-16/18 AS04-adjuvanted vaccine administered as a two-dose schedule in adolescent girls: Five-year clinical data and modeling predictions from a randomized study. Vaccine 12 (1):20-29

13. Immunization, Vaccines and Biologicals: Human papillomavirus (HPV) (2017).

14. Francis S, Katz M (2013) The HPV vaccine: a comparison of focus groups conducted in South Africa and Ohio Appalachia. Matern Child Health J 17 (7):1222-1229 [PubMed: 22930347]

15. Hertweck S, LaJoie A, Pinto M, Flamini L, Lynch T, Logsdon M (2013) Health Care Decision Making by Mothers for their Adolescent Daughters Regarding the Quadrivalent HPV Vaccine. Journal of Pediatric and Adolescent Gynecology 26 (2):96-101 [PubMed: 23518189]

16. Katz I, Nkala B, Dietrich J, Wallace M, Bekker L, Pollenz K, Bogart L, Wright A, Tsai A, Bangsberg D, Gray G (13) A qualitative analysis of factors influencing HPV vaccine uptake in Soweto, South Africa among adolescents and their caregivers. PLoS One 8 (8):e72094

17. Rambout L, Tashkandi M, Hopkins L, Tricco A (2014) Self-reported barriers and facilitators to preventive human papillomavirus vaccination among adolescent girls and young women: a systematic review. Prev Med 58:22-32 [PubMed: 24176938]

18. Oh J, Lim M, Yun E, Lee E, Shin H (2010) Awareness of and attitude towards human papillomavirus infection and vaccination for cervical cancer prevention among adult males and females in Korea: a nationwide interview survey. Vaccine 28 (7):1854-1860 [PubMed: 20005860]

19. Navarro-Illana P, Caballero P, Tuells J, Puig-Barbera J, Diez-Domingo J (2015) Acceptability of human papillomavirus vaccine in mothers from Valencia (Spain). Anales de pediatria 83 (5):318327 [PubMed: 25619797]

20. Watson-Jones D, Mugo N, Lee S, Mathai M, Vusha S, Ndirangu G, Ross D (2015) Access and attitudes of HPV vaccination amonst hard-to-reach populations in Jenya. PLoS One 10 (6):e0123701 [PubMed: 26115523]

21. Crann S, Barata P, Mitchell R, Mawhinney LTP, Chirenje M, Stewart D (2016) Healthcare providers' perspectives on the acceptability and uptake of HPV vaccines in Zimbabwe. Journal of Psychosomatic Obstetrics \& Gynecology 37 (4):147-155 [PubMed: 27400371]

22. Hopkins T, Wood N (2013) Female human papillomavirus (HPV) vaccination: Global uptake and the impact of attitudes. Vaccine 31 (13):1673-1679 [PubMed: 23375978]

23. Asiedu G, Breitkopf C, Kremers W, Ngo Q, Nguyen N, Barenberg B, Tran V, Dinh T (2015) Vietnamese health care providers' preferences regarding recommendation of HPV vaccines. Asian Pacific Journal of Cancer Prevention 16 (12):4895-4900 [PubMed: 26163611]

24. Holman DM, Benard V, Roland KB, Watson M, Liddon N, Stokley S (2014) Barriers to human papillomavirus vaccination among US adolescents: a systematic review of the literature. JAMA Pediatr 168 (1):76-82. doi:10.1001/jamapediatrics.2013.2752 [PubMed: 24276343]

25. McLellan E, MacQueen KM, Neidig JL (2003) Beyond the Qualitative Interview: Data Preparation and Transcription. Field Methods 15 (1):63-84

26. Vaismoradi M, Turunen H, Bondas T (2013) Content analysis and thematic analysis: Implications for conducting a qualitative descriptive study. Nursing and Health Sciences 15 (4):398-405 [PubMed: 23480423]

27. MacQueen KM, McLellan-Lemal E, Bartholow K, Milstein B (2008) Team-based Codebook Development: Structure, Process and Agreement. In: Handbook for Team-based Qualitative Researach. Altamina Press, Lanham, MD, pp 119-135

28. Bernard HR, Ryan GW (2010) Analyzing Qualitative Data: Systematic Approaches. Sage Publications Thousand Oaks, CA

29. Guest G, MacQueen KM, Namey EE (2012) Applied Thematic Analysis. Sage Publications Thousand Oaks, CA

30. Saldana J (2013) The Coding Manual for Qualitative Researchers (3rd ed.). Sage Publications, Thousand Oaks, CA

31. Jit M, Brisson M, Laprise J, Hong Choi Y (2015) Comparison of two dose and three dose human papillomavirus vaccine schedules: cost effectiveness analysis based on transmission model. BMJ 350 
32. Laprise J, Markowitz L, Chesson H, Drolet M, Brisson M (2016) Comparison of 2-Dose and 3Dose 9-Valent Human Papillomavirus Vaccine Schedules in the United States: A Costeffectiveness Analysis. The Journal of Infectious Diseases 214 (5):685-688 [PubMed: 27234416]

33. Gilkey MB, Calo WA, Moss JL, Shah PD, Marciniak MW, Brewer NT (2016) Provider communication and HPV vaccination: The impact of recommendation quality. Vaccine 34 (9): 1187-1192. doi:10.1016/j.vaccine.2016.01.023 [PubMed: 26812078]

34. Smith P, Stokley S, Bednarczyk R, Orenstein W, Omer S (2016) HPV vaccination coverage of teen girls: the influence of health care providers. Vaccine 18 (34):1604-1610

35. Rahman M, Laz T, McGrath C, Berenson A (2016) Provider recommendation mediates the relationship between parental human papillomavirus (HPV) vaccine awareness and HPV vaccine initiation and completion among 13- to 17-year-old U.S. adolescent children. Clinical Pediatrics 54 (4):371-375

36. Allison MA, Hurley LP, Marokwitz L, Crane LA, Brtnikova Michaela, Beaty BL, Snow M, Cory J, Stokley S, Roark J, Kempe A (2016) Primary care physicians' perspectives about HPV vaccine. Pediatrics 137 (2) e20152488 [PubMed: 26729738]

37. Kulczycki A, Qu H, Shewchuk R (2016) Primary Care Physicians' Adherence to Guidelines and Their Likelihood to Prescribe the Human Papillomavirus Vaccine for 11- and 12-Year-Old Girls. Women's Health Issues 26 (1):34-39 [PubMed: 26344447]

38. Kyong-No N, Chang K, Cho S, Park S, Park S (2017) Attitudes Regarding HPV Vaccinations of Children among Mothers with Adolescent Daughters in Korea J Korean Med Sci 32 (1):130-134 [PubMed: 27914142]

39. Vermandere H, Naanyu V, Mabeya H, Broeck D, Michielsen K, Degomme O (2014) Determinants of Acceptance and Subsequent Uptake of the HPV Vaccine in a Cohort in Eldoret, Kenya. PLoS One 10 (3):e0117761

40. Cunningham M, Skrastins E, Fitzpatrick R, Jindal P, Olola O, Yeates K, Booth C, Carpenter J, Aronson K (2015) Cervical cancer screening and HPV vaccine acceptability among rural and urban women in Kilimanjaro Region, Tanzania. BMJ Open 5 (3):e005828

41. Jaspers L, Budiningsih S, Wolterbeek R, Henderson F, Peters A (2011) Parental acceptance of human papillomavirus (HPV) vaccination in Indonesia: a cross-sectional study. Vaccine 29 (44): 7785-7793 [PubMed: 21821079]

42. Cover J, Nghi N, LaMontagne D, Huyen D, Hien N, Nga le T (2012) Acceptance patterns and decision-making for human papillomavirus vaccination among parents in Vietnam: an in-depth qualitative study post-vaccination. BMC Public Health 12 (629):629 [PubMed: 22877158]

43. Larson H, Jarrett C, Eckersberger E, Smith D, Paterson P (2014) Understanding vaccine hesitancy around vaccines and vaccination from a global perspective: A systematic review of published literature, 2007-2012. Vaccine 32 (19):2150-2159 [PubMed: 24598724]

44. Gallagher KE, Howard N, Kabakama S, Mounier-Jack S, Griffiths UK, Feletto M, Burchett HED, LaMontagne DS, Watson-Jones D (2017) Lessons learnt from human papillomavirus (HPV) vaccination in 45 low- and middle-income countries. PLoS One. 10.1371/journal.pone.0177773

45. Ferrer H, Trotter C, Hickman M, Audrey S (2014) Barriers and facilitators to HPV vaccination of young women in high-income countries: a qualitative systematic review and evidence synthesis. BMC Public Health 14 (700)

46. Galagan S, Paul P, Menezes L, LaMontagne D (2013) Influences on parental acceptance of HPV vaccination in demonstration projects in Uganda and Vietnam. Vaccine 31 (30):3072-3078 [PubMed: 23684835]

47. Widman C, Rodriguez E, Saad-Harfouche F, Tawrozek A, Erwin D, Mahoney M (2017) Clinician and Parent Perspectives on Educational Needs for Increasing Adolescent HPV Vaccination. Journal of Cancer Education:1-8 
- 2-dose

3-dose

- The same

Don't know/Not sure

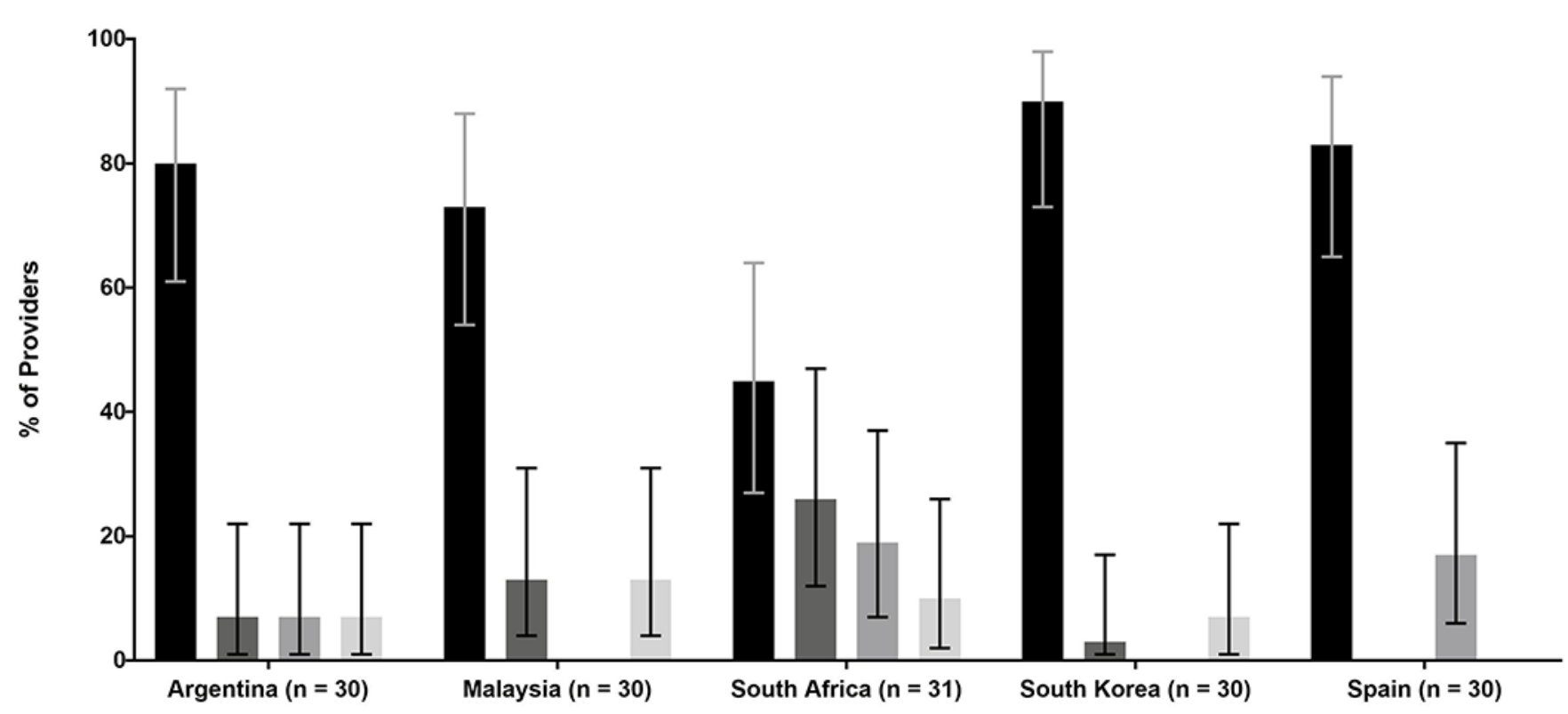

Figure 1:

If a 2-dose HPV vaccine were to become available, which would you recommend more frequently: A 3-dose HPV vaccine or a 2-dose HPV vaccine? 
$\square$ Fewer office visits

- More girls would complete series

Lower cost

Less pain

Fewer side effects

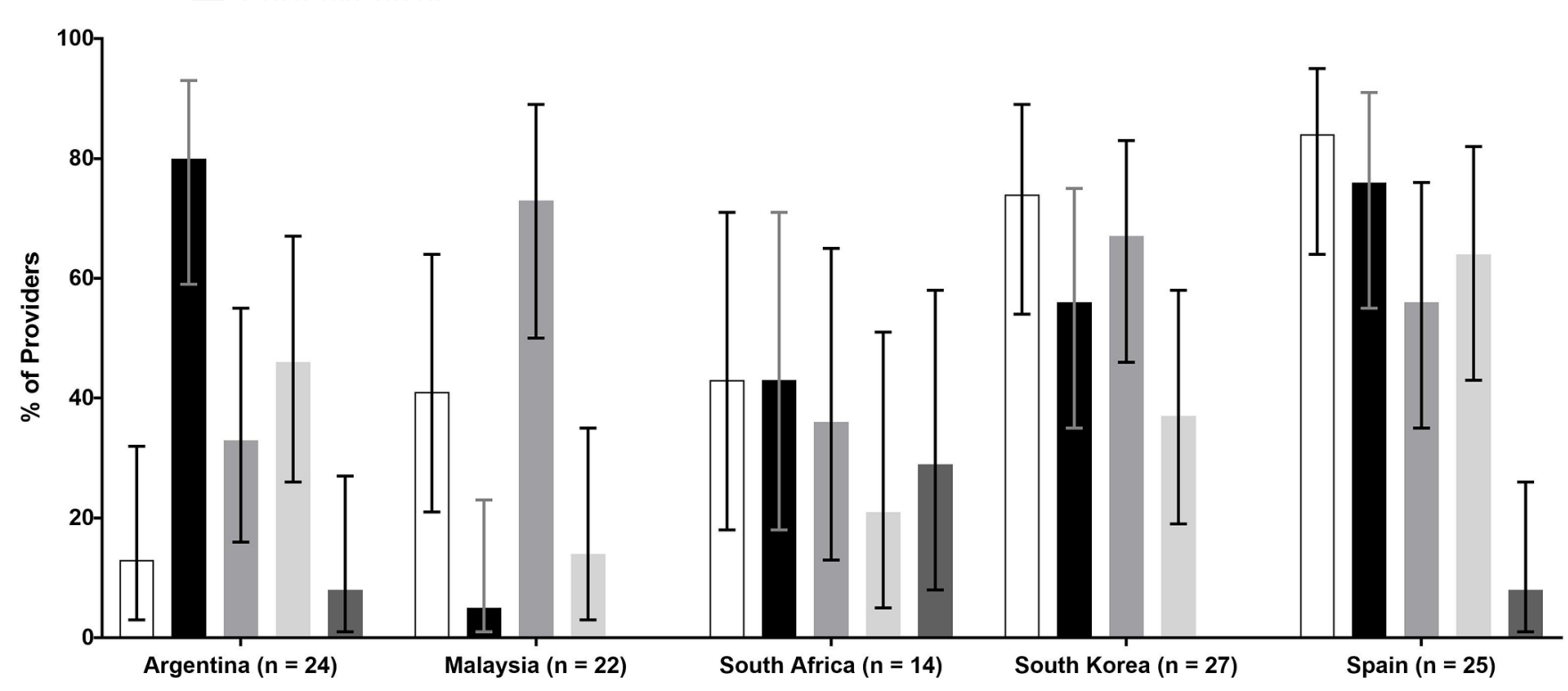

Figure 2:

[If 2-dose] Why would you recommend the 2-dose HPV vaccine more frequently to parents of girls?*

*Response may not add up to $100 \%$; Multiple responses possible. 


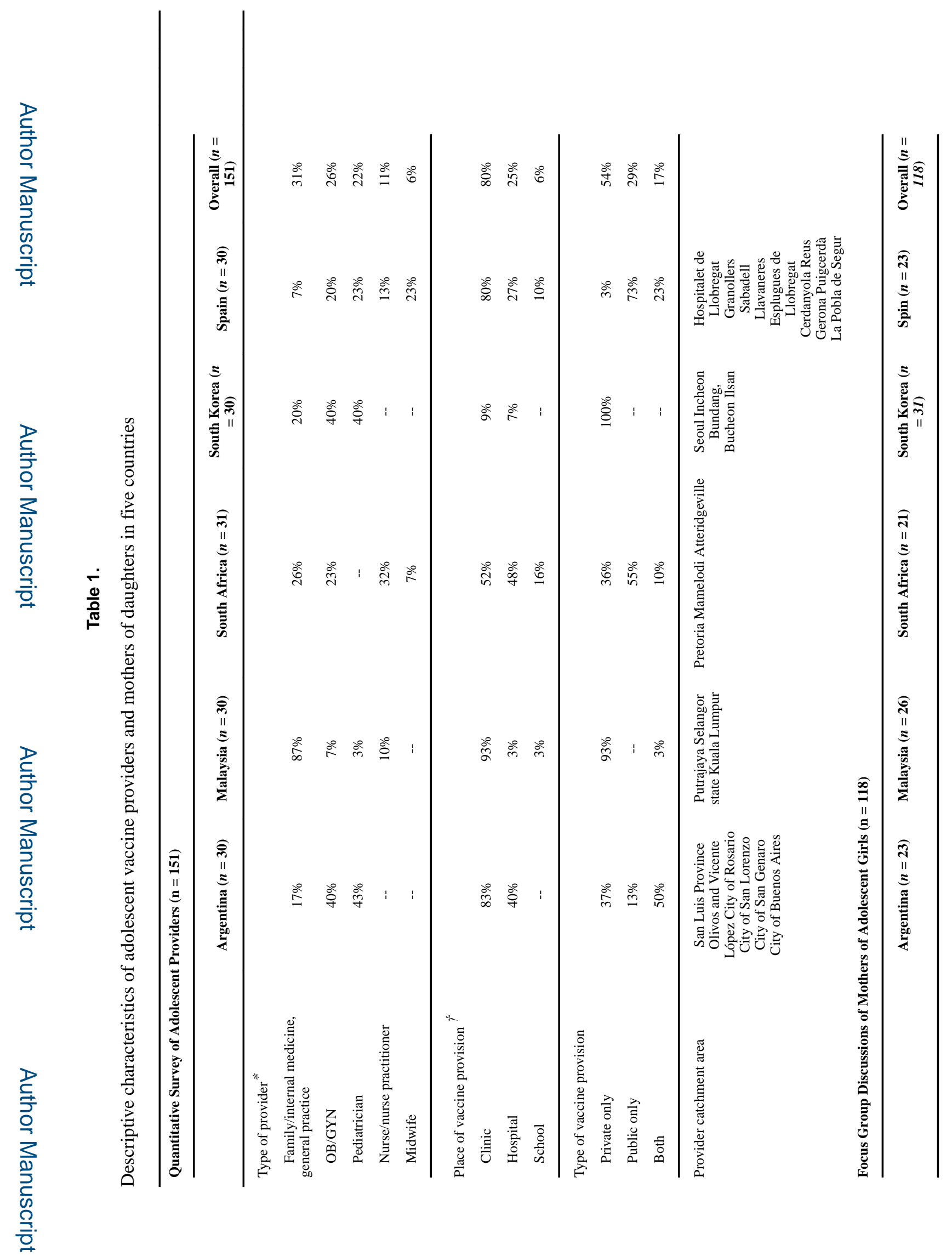

Cancer Causes Control. Author manuscript; available in PMC 2019 June 23. 


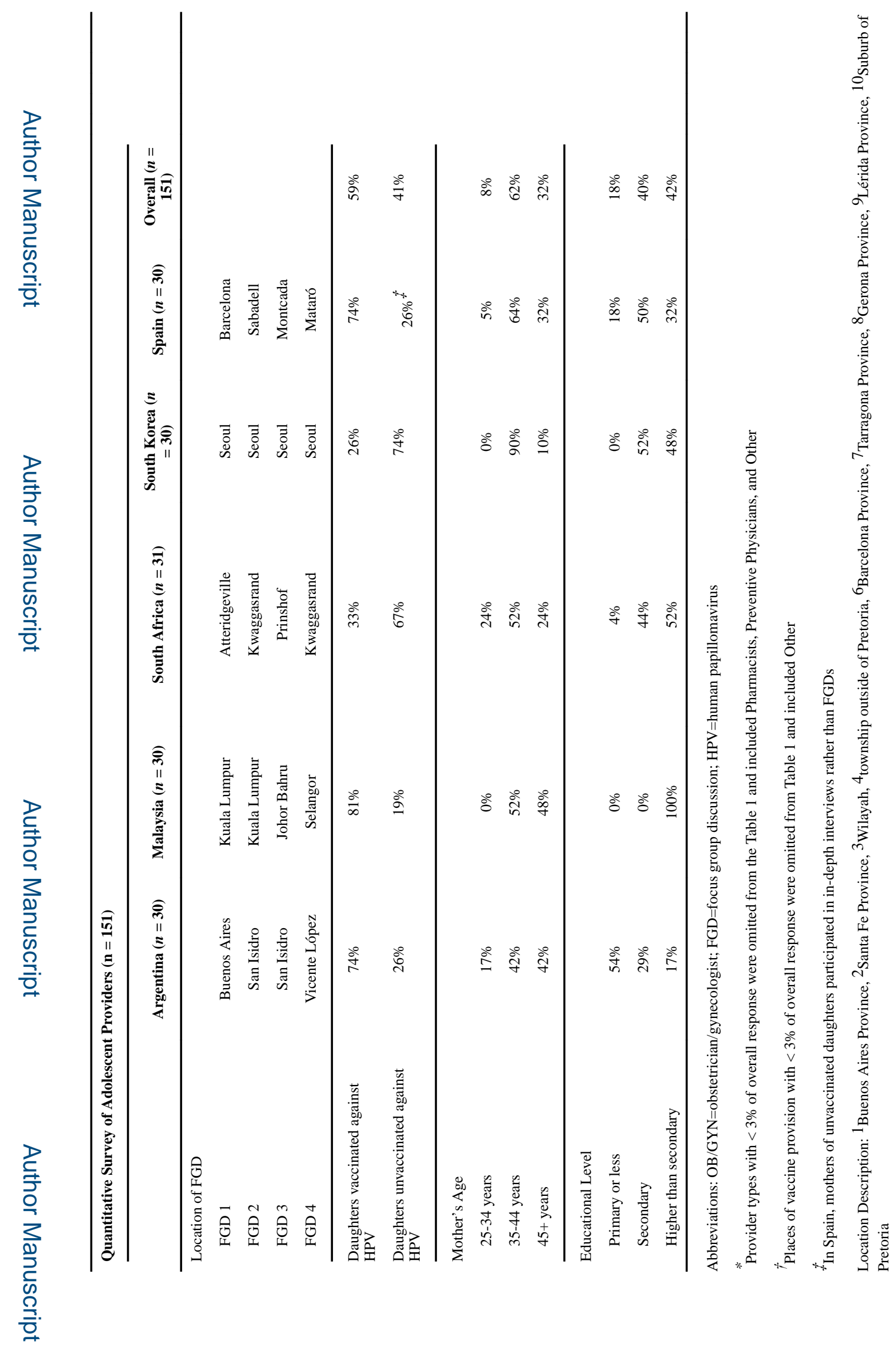

Cancer Causes Control. Author manuscript; available in PMC 2019 June 23. 


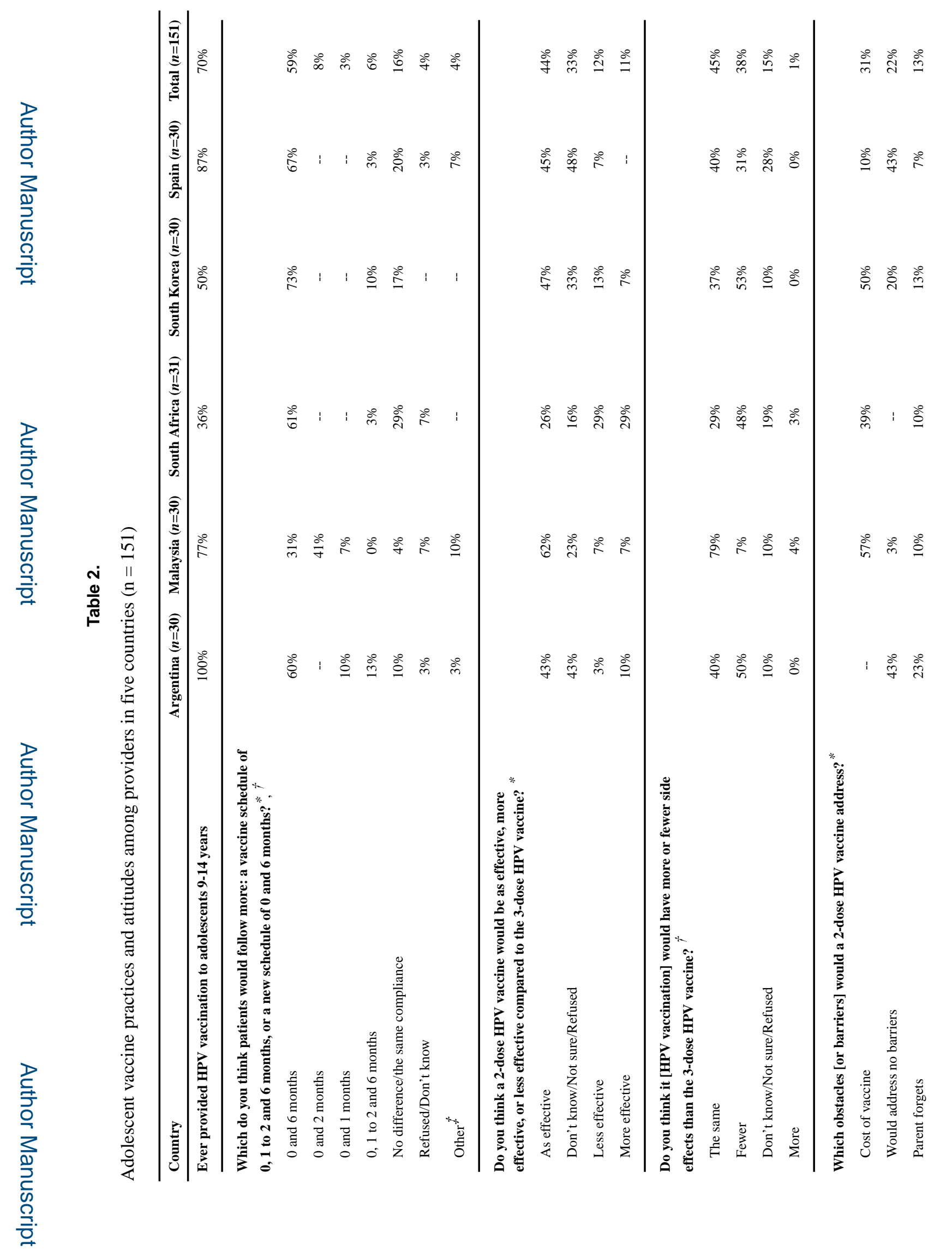

Cancer Causes Control. Author manuscript; available in PMC 2019 June 23. 
Islam et al.

Page 20

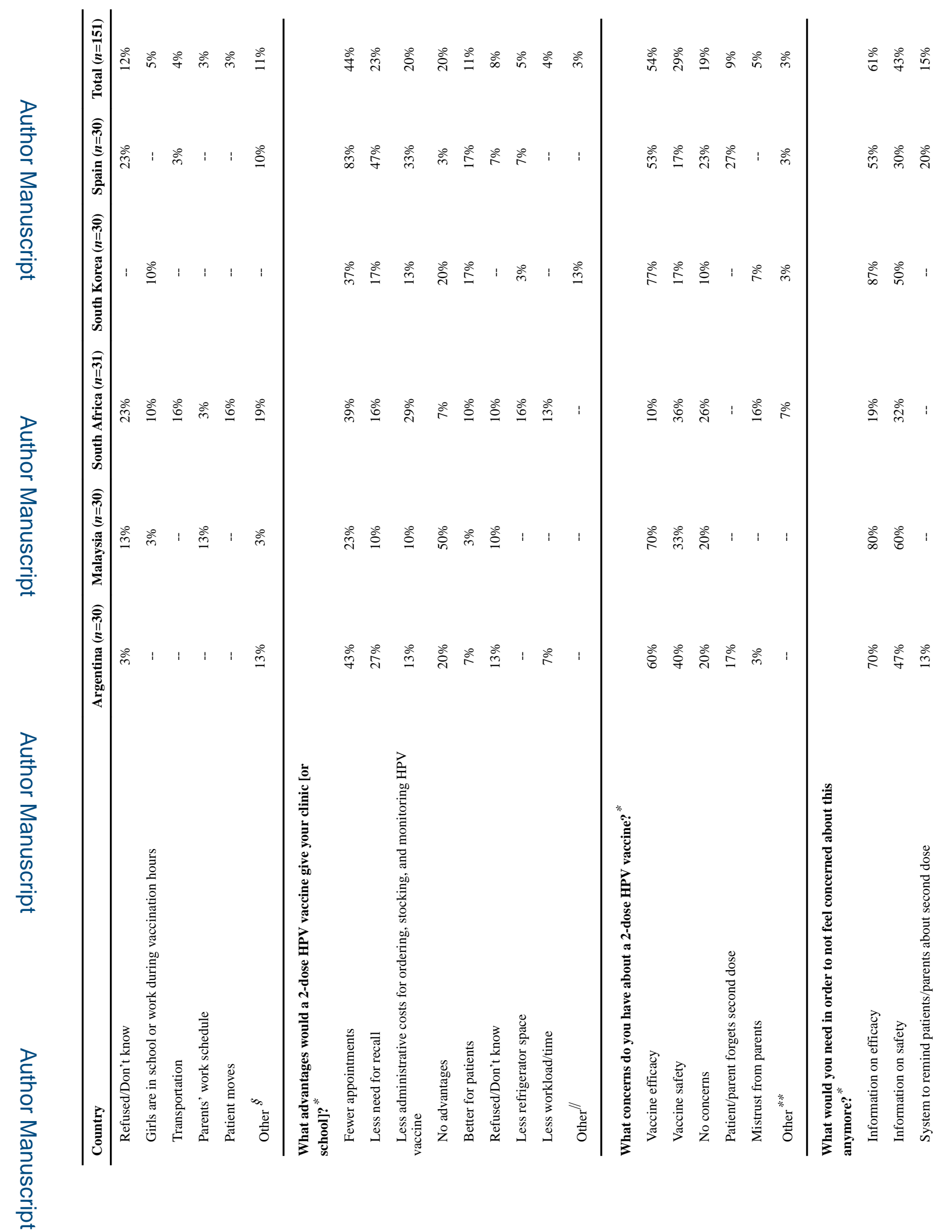

Cancer Causes Control. Author manuscript; available in PMC 2019 June 23. 
Islam et al.

Page 21

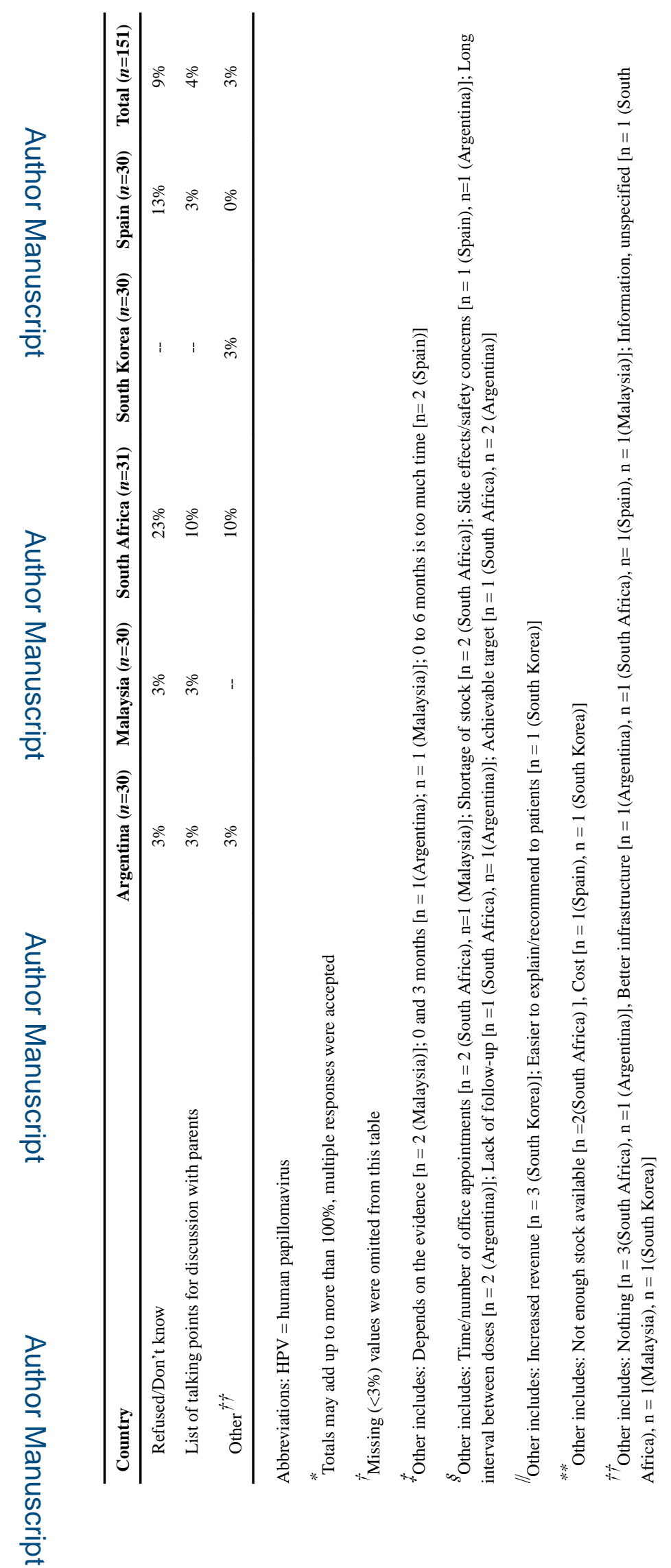

Cancer Causes Control. Author manuscript; available in PMC 2019 June 23. 


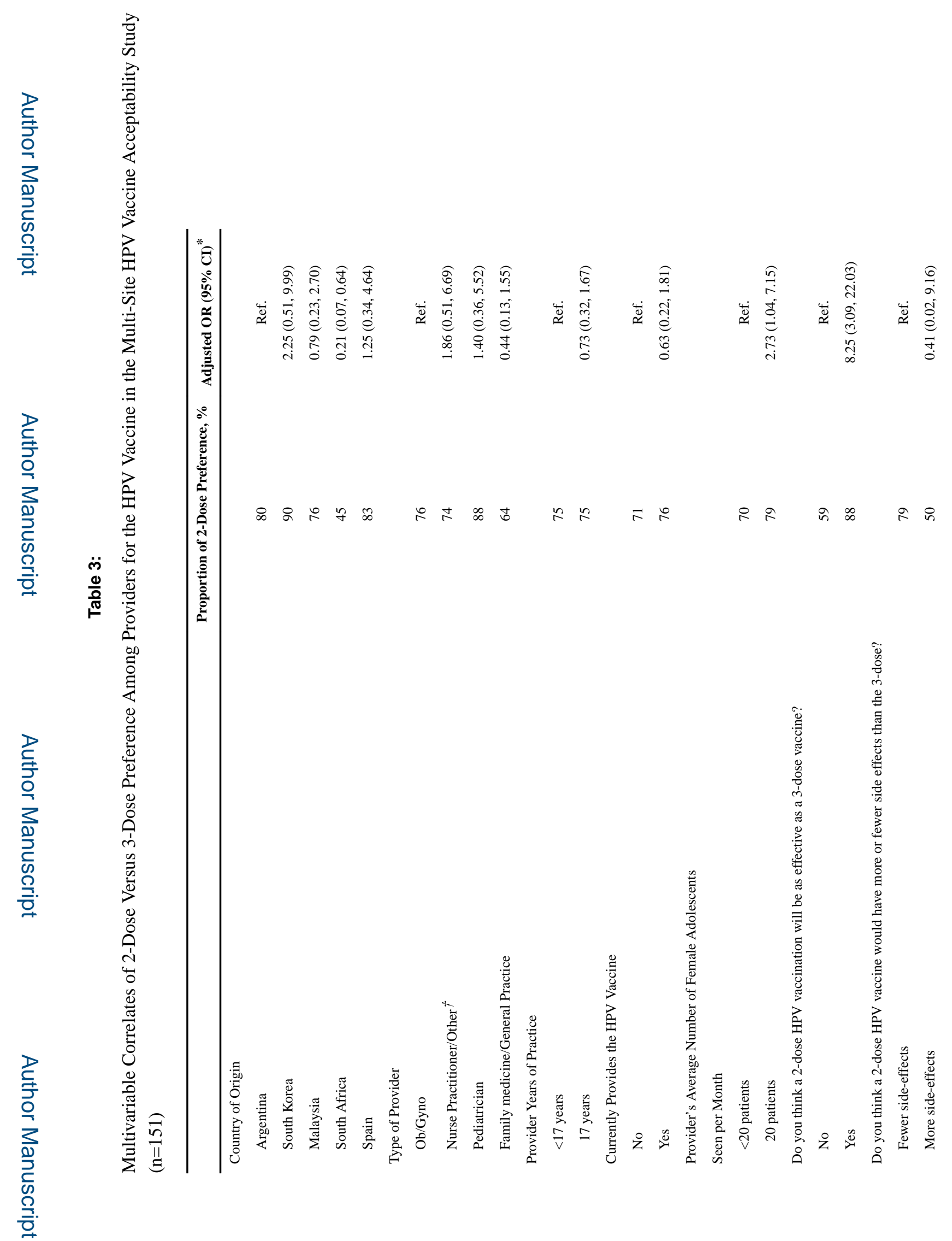

Cancer Causes Control. Author manuscript; available in PMC 2019 June 23. 


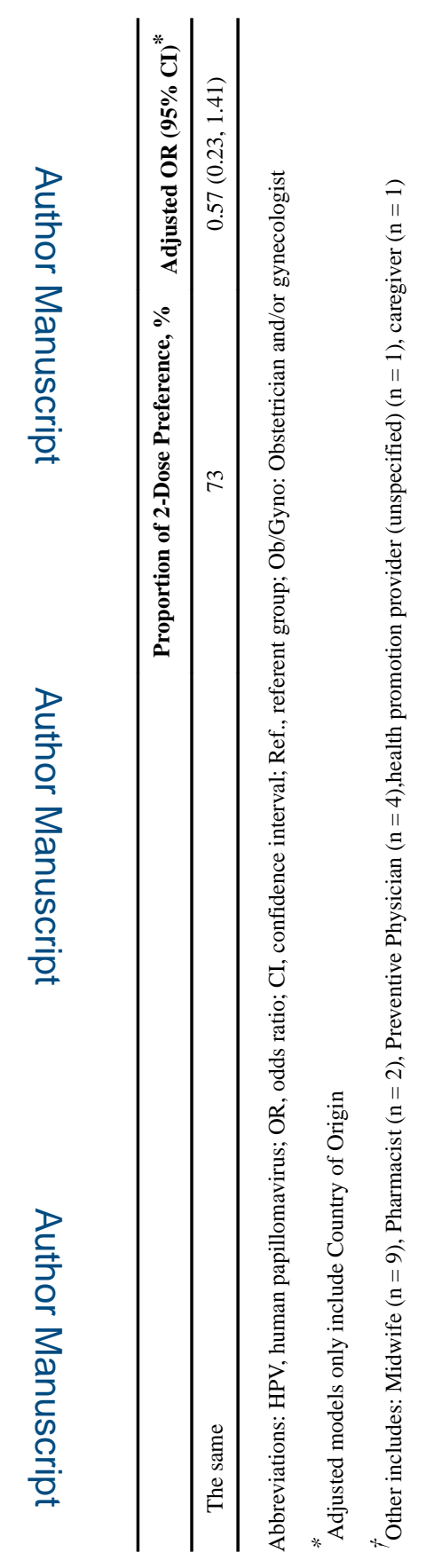

로을 


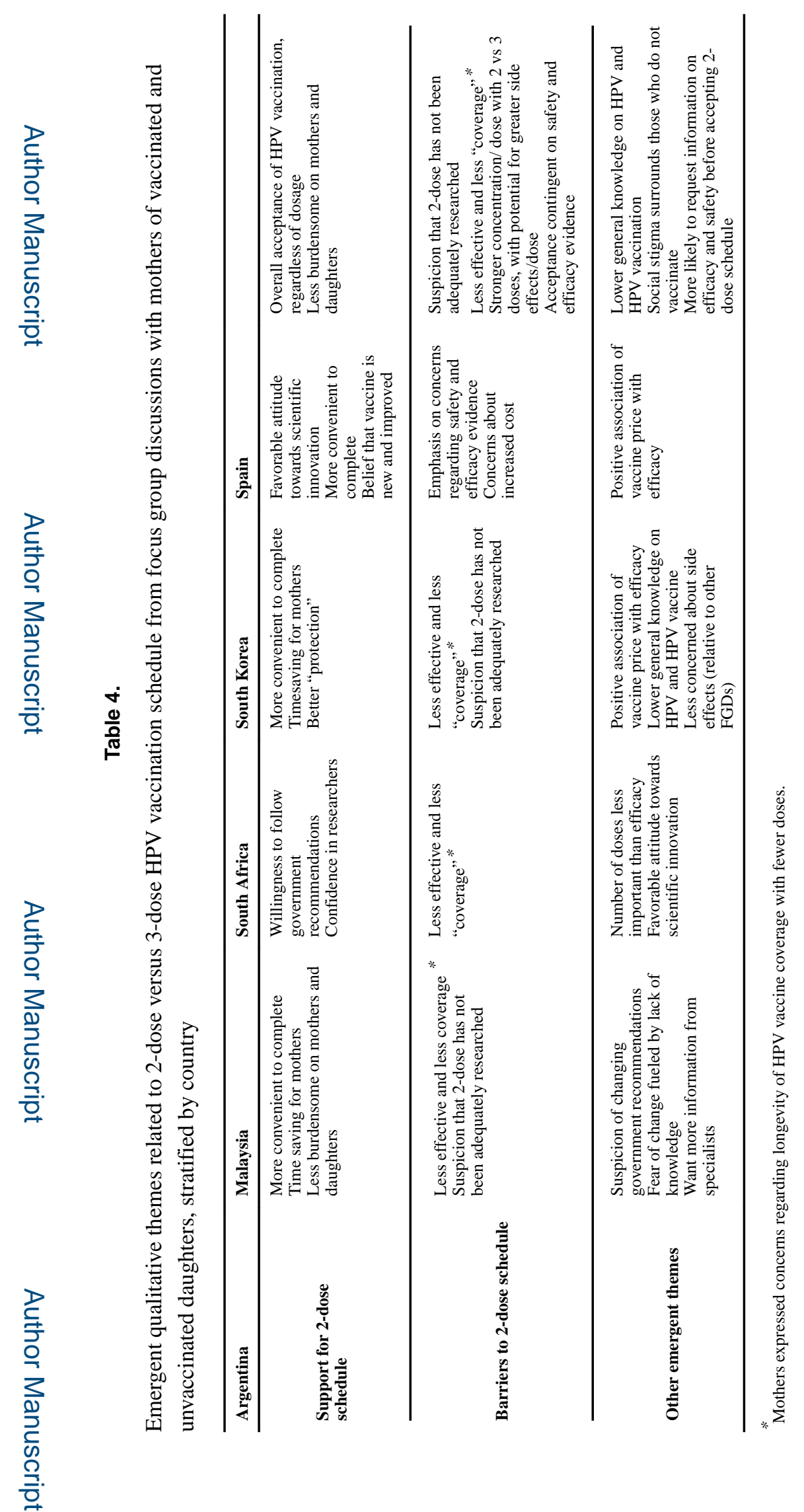

Cancer Causes Control. Author manuscript; available in PMC 2019 June 23. 